\title{
Toward More Precise Beam Positron Measurements
}

\author{
T.J. Fessenden \\ J.C. Clark \\ Cliff Holmes
}

May 12, 1999

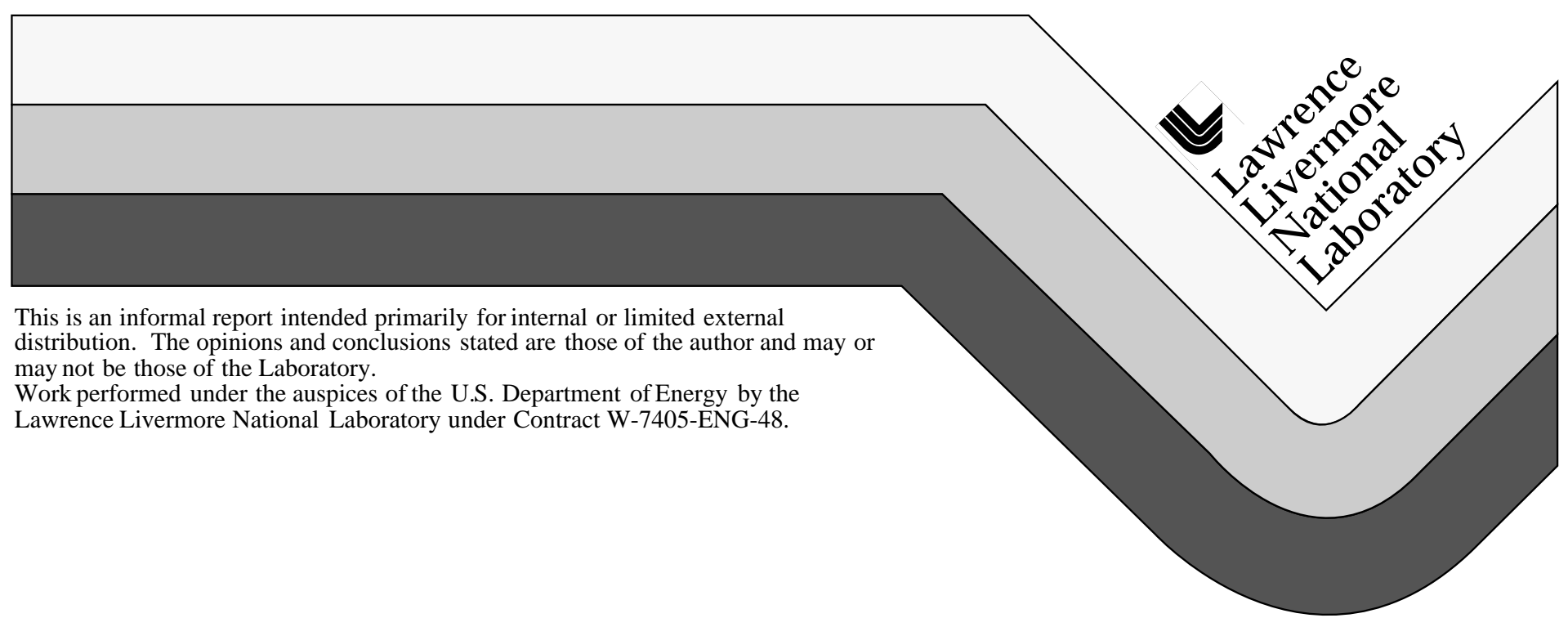




\section{DISCLAIMER}

This document was prepared as an account of work sponsored by an agency of the United States Government. Neither the United States Government nor the University of California nor any of their employees, makes any warranty, express or implied, or assumes any legal liability or responsibility for the accuracy, completeness, or usefulness of any information, apparatus, product, or process disclosed, or represents that its use would not infringe privately owned rights. Reference herein to any specific commercial product, process, or service by trade name, trademark, manufacturer, or otherwise, does not necessarily constitute or imply its endorsement, recommendation, or favoring by the United States Government or the University of California. The views and opinions of authors expressed herein do not necessarily state or reflect those of the United States Government or the University of California, and shall not be used for advertising or product endorsement purposes.

This report has been reproduced directly from the best available copy.

Available to DOE and DOE contractors from the Office of Scientific and Technical Information P.O. Box 62, Oak Ridge, TN 37831

Prices available from (423) 576-8401

Available to the public from the National Technical Information Service

U.S. Department of Commerce 5285 Port Royal Rd. Springfield, VA 22161 


\section{Toward More Precise Beam Position Measurements T.J. Fessenden, J.C. Clark, Cliff Holmes \\ May 12, 1999}

\section{Introduction}

For the past year or so we have been examining the properties and limitations of the beam bugs in use in the ETA program at LLNL with a view toward improving the accuracy of beam position and current measurements.

When considering measurements of beam position, it is very important to distinguish between relative and absolute position measurements.

A relative position measurement determines only the amplitude and direction of the motion of the beam within the transport tube. If one knew where the beam was, one could determine its new position. A relative measurement is essentially independent of errors in mechanical fabrication or electrical components. The minimum measurable displacement is only limited by the strength of the electrical signals or the signal to noise ratio of the position signal.

An absolute position measurement is much more challenging. All inaccuracies in mechanical components and fabrication, electrical components, installation and assembly errors must be considered and controlled along with the issues common to relative position measurements. However, if the object is to strike a small specific point on a target or pass the beam through a small hole, absolute beam position measurements are required.

The following is a summary of our progress including conclusions and recommendations for developments and improvements. This is, of course, only a step in beam bug development and there is plenty of room for others to contribute.

\section{Physics Issues}

\section{Theory overview}

As shown in several references the voltage distribution around the circumference of a beam bug generated by a beam offset from the system axis by a normalized amount $\rho$ is given by

$$
V(\theta)=K(\theta) 2 \pi a R=R I \frac{1-\rho^{2}}{1+\rho^{2}-2 \rho \cos \theta} .
$$

Here $\rho$ is the actual beam displacement divided by the pipe radius a; $R$ is the total shunt resistance of the bug and $\theta$ is the angle from the $\mathrm{x}$-axis to the beam. The ETA bug signal is developed by picking off this voltage at the four cardinal points around the bug and adding all to form the current signal and subtracting opposite pairs to generate the $\mathrm{x}$ and $\mathrm{y}$ position signals. 


\section{Number of pickoffs}

For measurements of the position of off-axis beams, eight or more pickoffs are necessary. Position measurements generated by only four pickoffs are subject to large errors for relatively small beam displacements from axis. Four pickoffs are perfectly adequate for detecting off-axis beams and guiding the operator toward tuning them to the axis. However, off-axis beam position measurements require at least 8 pickoffs. This point is illustrated in figure 1. Here are plotted the ratios of measured to actual beam offset versus the actual beam offset for four and eight pickoffs around the bug circumference. These results were developed from the above equation. The curves in each case bound the values generated by any beam displacement angles $\theta$. That is, depending on the angle $\theta$, the measurement can fall anywhere between the two curves as a function of $\rho$. As an example a beam offset by half the distance to the wall will be subject to an error of $\pm 25 \%$ using 4 pickoffs but only to an error of $\pm 2 \%$ using 8 pickoffs.

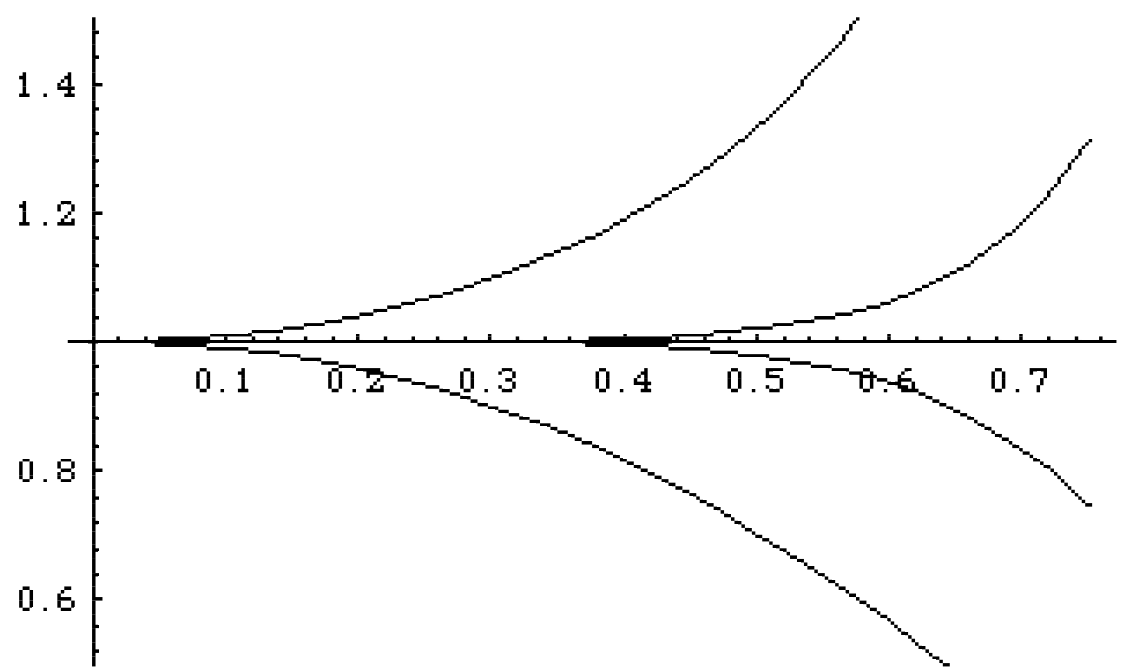

Fig. 1. Plot of the measured/actual versus actual offset $\rho$ using 4 and 8 pickoffs. The errors at different angles are bounded by the curves.

As an added bonus, errors generated during beam current measurements are bounded at all angles by $1 \pm 2 \rho^{n}$ where $\mathrm{n}$ is the number of pickoffs. For a beam half way to the wall, the current measurement can be in error by as much as $\pm 12.5 \%$; with 8 pickoffs this error is limited to $\pm 0.78 \%$.

\section{Mechanical Issues}

A set of experiments was performed using the beam bug test stand. In these experiments the center conductor was centered and the signals generated at the eight output ports were carefully measured. Ideally, these signals would all have been equal. Experiments showed that the signals varied in amplitude by as much as $\pm 1.5 \%$.

Experiments traced the cause of these variations to primarily two effects. The first is variations in the foil thickness supplied by the vendor; the second is variations in the spot welds around the inner circumference of the bugs. Results of the experiments are presented in Fig. 2. 


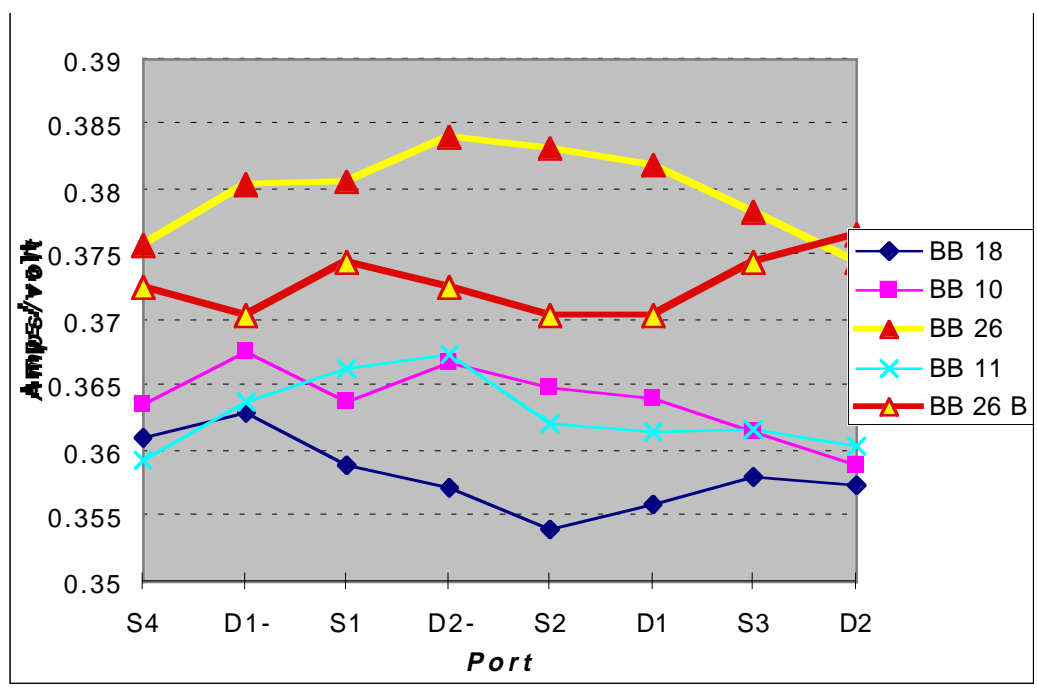

Fig 2a. Absolute signal outputs.

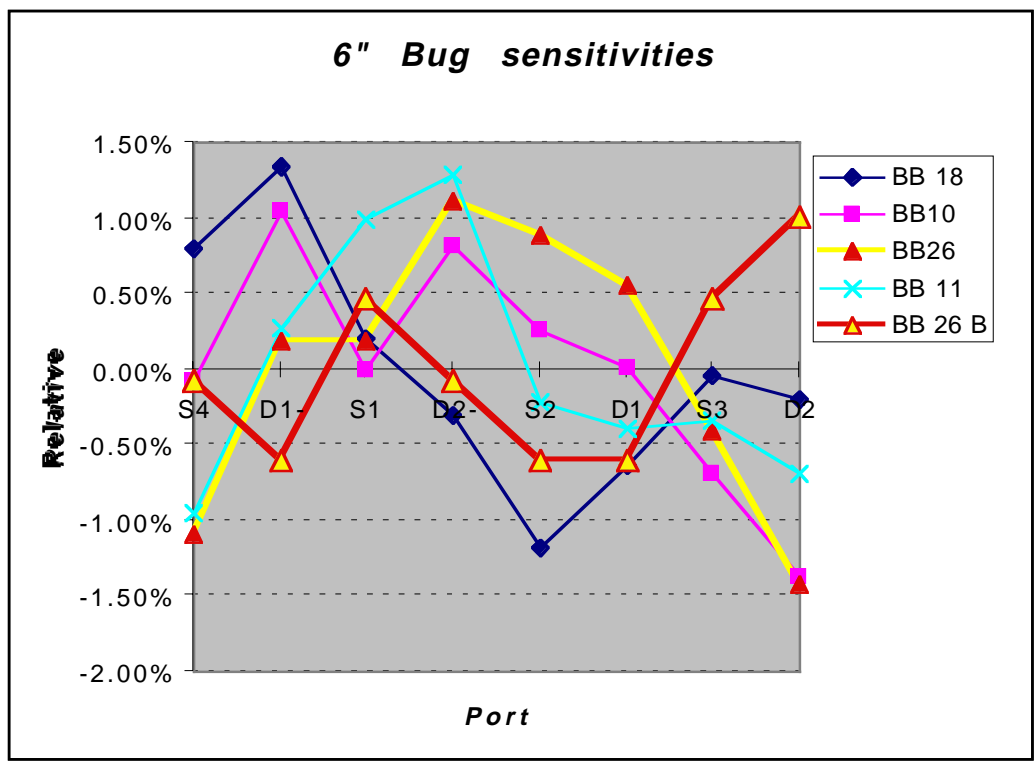

Fig 2 b. Relative signal outputs.

Fig. 2. Plots of the absolute and relative variations of the outputs of four ETA beam bugs at the 8 output ports. BB 26 was measured with two different foils.

For the initial experiments the foil on BB 26 was crinkled due to vacuum loading. A new foil was very carefully reattached to beam bug 26 by one of us (Cliff Holmes). Care was also taken to minimize the foil overlap. Data gathered from the refoiled bug 26 is labeled BB 26 B in figure 2 above. These tests showed that extra care in attaching the foil did reduce the variation in port signal amplitude but by less than half.

\section{Foil resistance variation}




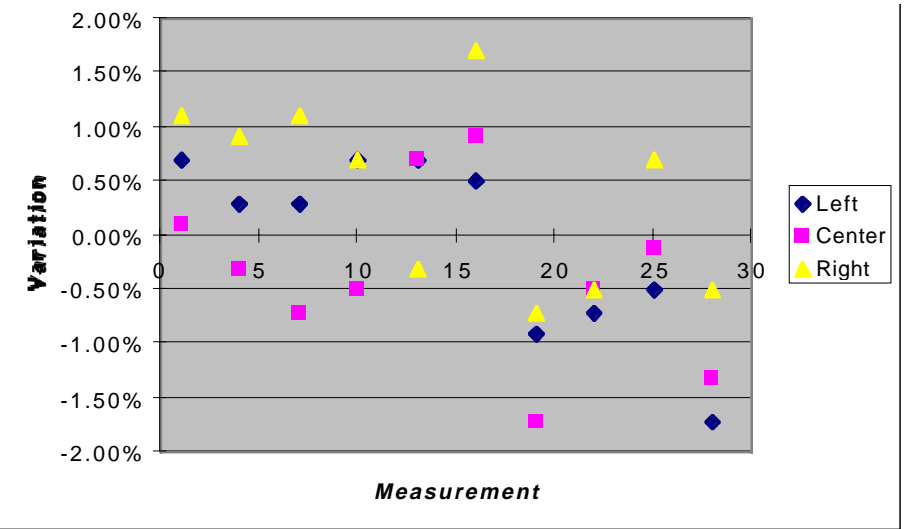

The foil used on the ETA II bugs is 0.2-mil nichrome. This is a thickness of only $5.08 \mu \mathrm{m}$ ! The thickness of unused samples of the 0.2-mil nichrome foils was measured

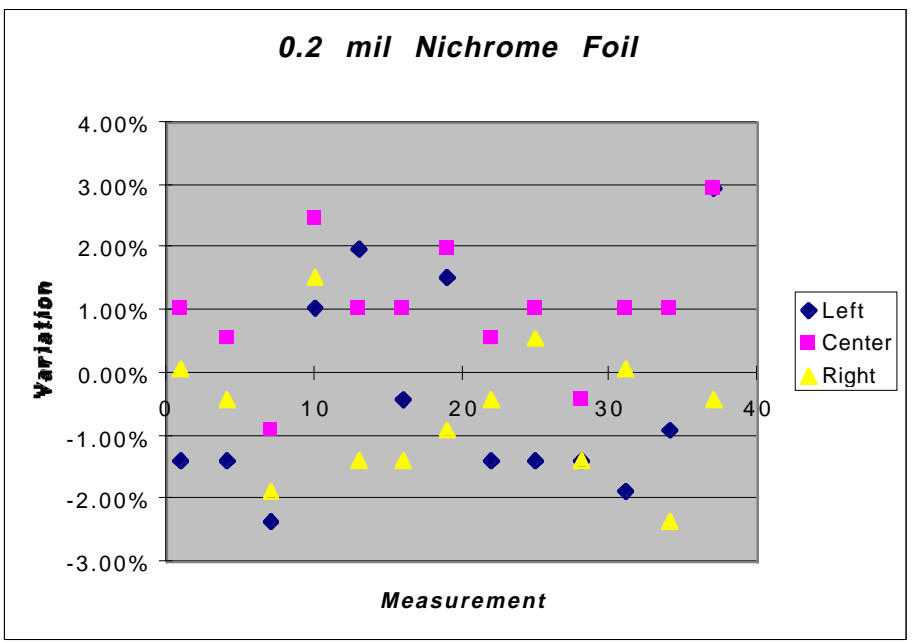

using a laser interferometer at many places on the foil. Measurements were believed accurate to \pm 1 microinch! Results are shown in Fig 3. These measurements revealed thickness variations of about $\pm 2 \%$. About the same as the variations in the output of the bug ports mentioned above. A second set of measurements using 0.5-mil stainless steel foil showed a similar percentage variation. The measurements in each test were checked for repeatability. Conversations with the manufacturer suggested that $a \pm 2 \%$ variation in foil thickness is as good as can be expected by the rolling mill. We conclude that much of the variation in output port signal is attributable to this variation in foil resistance.

Fig. 3. Measurements of the variation of the thickness of fresh 0.2-mil Nichrome and 0.5-mil Stainless foils. The foils were 2 inches wide. In each foil approximately 40 measurements were taken toward the left, center, and right side of the foils.

\section{Foil crinkling}

Repeated vacuum loads on beam bugs installed in a beam transport line cause the foils to crinkle and distort. This crinkling is less detrimental to position measurements than one might suspect. Nevertheless, it should be reduced or eliminated if possible. Pumpout holes around the foils or even many holes in the foils themselves might eliminate the source of the crinkling. More work is needed. 


\section{The foil overlap problem}

The resistor in each beam bug is formed by welding either a nichrome or stainless steel foil across an insulator in the outer circumference of the beam bug. As a consequence either an overlap or underlap of length $\Delta$ is created. For the case of an underlap the resistance is very high for the length $\Delta$ and all the bug signals will be very noisy. An overlap eliminates this noise and is greatly to be preferred. Nonetheless, for the length $\Delta$ the circular resistance will be one half nominal leading to a small error in the measurement of beam position.

The overlap problem was analyzed in considerable detail and is presented in references 2 and 3 . There it is shown that the overlap effectively limits the smallest beam offset that can be measured to approximately $\Delta / 4 \pi$ independent of the radius of the bug. For a typical overlap of 10 mils the error introduced is only about one mil. This analysis assumes that the overlap is many $\Delta$ s away from a pickoff point.

\section{Electrical Issues}

The ETA bugs use a 0.2-mil nichrome foil as the resistive shunt and are designed to maximize the current and position signal. As a consequence, during beam operations, $10-\mathrm{X}$ attenuators are customarily used to match the signals into the oscilloscope. A further consequence of this high resistance foil is that the droop of the position signal is only $200 \mathrm{~ns}$. That means that a perfectly stable off-axis beam appears to sweep about $1 / 3$ the offset distance toward the axis in the time of a $70 \mathrm{~ns}$ ETA pulse. The computer program that is used to develop the beam position is "aware" of this and conditions the signal using the following algorithm.

$$
V_{p}(n)=V_{s}(n)+\frac{\Delta t}{t_{d}} \sum_{i=1}^{n} V_{s}(i)
$$

Here $V_{p}(n)$ is the corrected position signal; $V_{s}(n)$ is the position signal from the bug; $\Delta t$ is time measured from the start of the beam; $t_{d}$ is the position signal droop time. This technique works best when the beam pulse width is less that the droop time $t_{d}$ Experiments showed that this technique could not be used to extend position measurements using ETA II bugs to the $2 \mu$ s DHRT pulse.

\section{Beam Bugs for DAHRT}

The present mechanical design can be adopted for DAHRT by using the present bug cross-section design and adjusting the bug diameter as required. The principal change recommended is simply to replace the present 0.2 -mil nichrome foil with 1.0 or 2.0 mil stainless foil. This would reduce the signal voltages generated by the DAHRT beam by a factor of 5-10. There are several reasons for this recommendation.

1) The present ferrite cross-section is about 10 times too small for the $2-\mu \mathrm{sec}$ DAHRT pulse. At full DAHRT current, the signal voltage can be expected to saturate the ferrite within $2 \mu \mathrm{sec}$. Either the ferrite cross-section must be 
increased by about a factor of ten or the voltage generated by the foil must be correspondingly reduced.

2) Bench tests show that the present droop time for the current signal is approximately $20 \mu \mathrm{sec}$. For the $100 \mathrm{~ns}$ ETA II pulse the pulse droop is $0.5 \%$. For the DAHRT pulse this droop would amount to approximately $10 \%$ which is unacceptable. Decreasing the foil resistance would reduce the droop to about $1 \%$. (Increasing the ferrite area by ten would have the same effect).

3) The present droop time for the position signal is approximately $0.2 \mu \mathrm{sec}$. This droop must be compensated either passively or through computer processing. Our tests on a standard ETA bug showed that this compensation was difficult with pulses as long as $2.0 \mu \mathrm{sec}$. Reducing the foil resistance by ten would extend this droop time to $2.0 \mu \mathrm{sec}$ and greatly facilitate the compensation.

4) One of the sources of error in the measurement of beam position is generated by the spot welding of the 0.2 -mil nichrome foil to the stainless steel bug housing. Past tests have revealed that this operation contributes at least $1 \%$ to the error in beam position measurements. The thicker 1.0 mil stainless foil can be laser welded to the housing, an intrinsically more precise operation. Laser welding should reduce the error generated in attaching the resistive foil to the stainless housing.

On the negative side the lower resistance foil will reduce the voltage level of the signals. This should not affect the utility of the bugs for measuring the kiloampere DAHRT beam but may create an issue when calibrating the bugs in the test setup with 20 Ampere test pulses.

Scaling the bug from the present nominal 6-inch diameter to 10 or 14 inch diameter will have the following consequences (see for example Reference 1):

The resistance of the bug and the signal voltages will scale inversely with the beam diameter. Since the bug measures beam position normalized to the bug radius, errors in the measurement of beam position will also scale with the bug diameter. The accuracy of beam position measurements is approximately $\pm 2 \%$. If so, the absolute error in beam position measured with a 14-inch diameter beam bug should be near $\pm 4 \mathrm{~mm}$.

- For the same axial length and thickness foil, the droop of the current and position signals scale as the square of the bug diameter. (The inductance scales directly and the foil resistance inversely as the bug diameter.)

One last comment. The major expense in bug fabrication is machining the stainless steel bug housings. It may be possible to reduce this cost by value engineering the design. On the other hand, depending on the number of bugs to be fabricated, the cost of this engineering may not be recovered. 


\section{Precise Beam Position Measurements}

Using 8 pickoffs the absolute position of off-axis beam should be measurable to approximately $\pm 1 \%$ of the pipe radius. For a 10-inch diameter pipe the error is \pm 50 mils or $\pm 1.25 \mathrm{~mm}$. This error is 10 or more times larger than permissible for guiding beams through 5 mil holes. Relative position measurements can be made with much greater precision. Therefore, if greater precision is required in situ calibration may be the solution.

Two thoughts come to mind. The first is a test setup utilized as the bug is installed. This could consist of a conducting rod centered and connected to the position of interest. A fast current pulse through the rod could provide reference signals for the beam bug. The absolute beam position could be determined by relative measurements using these reference signals. A second approach is to use the beam itself properly attenuated. The reference signals might be obtained by maximizing the current to a detector downstream of a small hole at the position of interest. The beam must be comparable in size to the hole and the beam current reduced to the point that downstream components are not damaged. Possibly, this necessarily reduced current will be too small for generating adequate reference signal.

\section{Conclusions and Summary}

1) For the measurement of the position of off-axis beams, at least eight-pickoffs are required.

2) Variations in the thickness of the foils are a major contributor to errors in beam position measurements using beam bugs. This error in itself limits absolute measurements of beam position to at least $\pm 1 \%$. This variation is essentially the same for 0.2 mil nichrome and 0.5 mil stainless foils. It is possible but not likely that 1.0 mil or thicker stainless foils will have a smaller percentage variation.

3) Spot welding of the foil to the bug generates an error of the same order as the variation in foil resistance. A process using laser welding should reduce this error.

4) Foil overlap leads to a negligible error in the measurement of beam position if the overlap is limited to about 10 mils and all the bug pickoffs are located 0.1 inch or more away from the overlap.

5) ETA beam bugs can be adapted to measure both the current and position of $2 \mu \mathrm{sec}$ beam pulses relevant to the DAHRT mission. The major modification required is to reduce the foil resistance by a factor of at least 5 . This can be easily accomplished by using 1.0 mil or 2.0 -mil stainless foil.

6) It will be extremely difficult to measure absolute beam positions to accuracies less than $\pm 1 \%$ of the bug radius. Better precision may be possible with a careful in situ calibration of the beam bug at critical locations. 


\section{References}

1 Fessenden, Stallard, and Berg, RSI 43, p.1789 (1972)

2 T.J. Fessenden, "Beam Bugs - Asymptotic Response," Beam Research Memo BM 88-8, (1988).

3 T.J. Fessenden, "Diagnostics for Induction Accelerators," Proceedings of the Beam Instrumentation Workshop, Argonne National Laboratory (1996). 